Int. J. Speleol. 10 (1978), pp. $245-252$

\title{
FRACTURATION ET KARSTIFICATION D'UN MASSIF: L'EXEMPLE DE L'AZEROU EL KEBIR (Algérie du Nord)
}

\author{
par \\ P.E. COIFFAIT* et Y. QUINIF**
}

Fracturation and Karstification of a Massif: the exemple of the Azerou el Kebir (Northern Algeria)

SUMMARY

The fracturation of the sub-autochthonous massif of the Azerou el Kebir is not fundamentally different from that of the adjoining allochthonous massif - where the structures are due to an $\mathrm{Al}$ pine phase, known as the Atlas phase. As with all fractures, karstification only exploits certain of them, without having any linkage to their statistical importance, caves have developed following fractures which are qualitatively important, but are poorly represented quantitatively. The study of the karstification therefore, confirms his complementary to the structural analysis in order to elucidate the technical problems of fracturation in the region.

\section{INTRODUCTION}

Le massif calcaire de l'Azerou el Kébir (Fig. 1), bordant au Sud la chaîne des Bibans, juste après les portes de fer, correspond à un pointement à travers les nappes telliennes (Caire, 1957) du substratum carbonaté affleurant en série inverse (Coiffait, Vila, 1977). L'étude de sa karstification a abouti à la reconnaissance et à la topographie de la grande grotte (Coiffait, Quinif, 1976, 1977). Le plan de cette grotte montre trois grandes directions de fissures verticales ou subverticales exploitées par la karstification (Fig. 2).

Devant cette particularité, nous avons entrepris l'étude de la fracturation de l'Azerou el Kébir proprement dit aux environs immédiats du domaine karstifié. Nous avons ensuite comparé cette fracturation à celle des nappes, toujours à proximité de la grotte. Enfin, nous avons tenté de voir l'origine de la fracturation des nappes en étudiant l'anticlinal qu'elles forment.

(*) Institut des Sciences de la Terre et C.U.R.E.R., Université de Constantine (Algérie) et Laboratoire Associé C.N.R.S. $n^{\circ}$ 145: Géologie méditerranéenne.

$\left(^{* *}\right)$ I aboratoire de Minéralogie et de Pétrographie, Faculté Polytechnique de Mons, rue de Houdain, 7000 Mons (Belgique). 
Fig. 1: Carte géologique et localisation de l'Azerou el Kébir.

Terrains récents:

1) Quaternaire indifférencié (alluvions, dépôts de pente, terrasses, placages);

2) travertins.

\section{Terrains allochtones:}

3) Sénonien: marnes grises alternant avec des petits bancs de calcaires clairs, bien lités (lame A4 de A. CAIRE);

4) Paléocène: marnes sombres avec des petits bancs calcaires assurant un passage très progressif aux calcaires sus-jacents;

5) Yprésien - Lutétien inférieur: calcaires lités à silex (forment avec les marnes paléocènes la lame A3 de A. CAIRE).

Terrains sub-autochtones:

6) Albien supérieur - Vraconien: marnes et marno-calcaires;

7) Cénomanien inférieur: dolomie massive à patine fauve;

8) Cénomanien au moins moyen: calcaire blanc biodétritique à Rudistes et Orbitolines.

On notera le sub-autochtone en série inverse perçant un allochtone en série normale et débité en lames indépendantes.

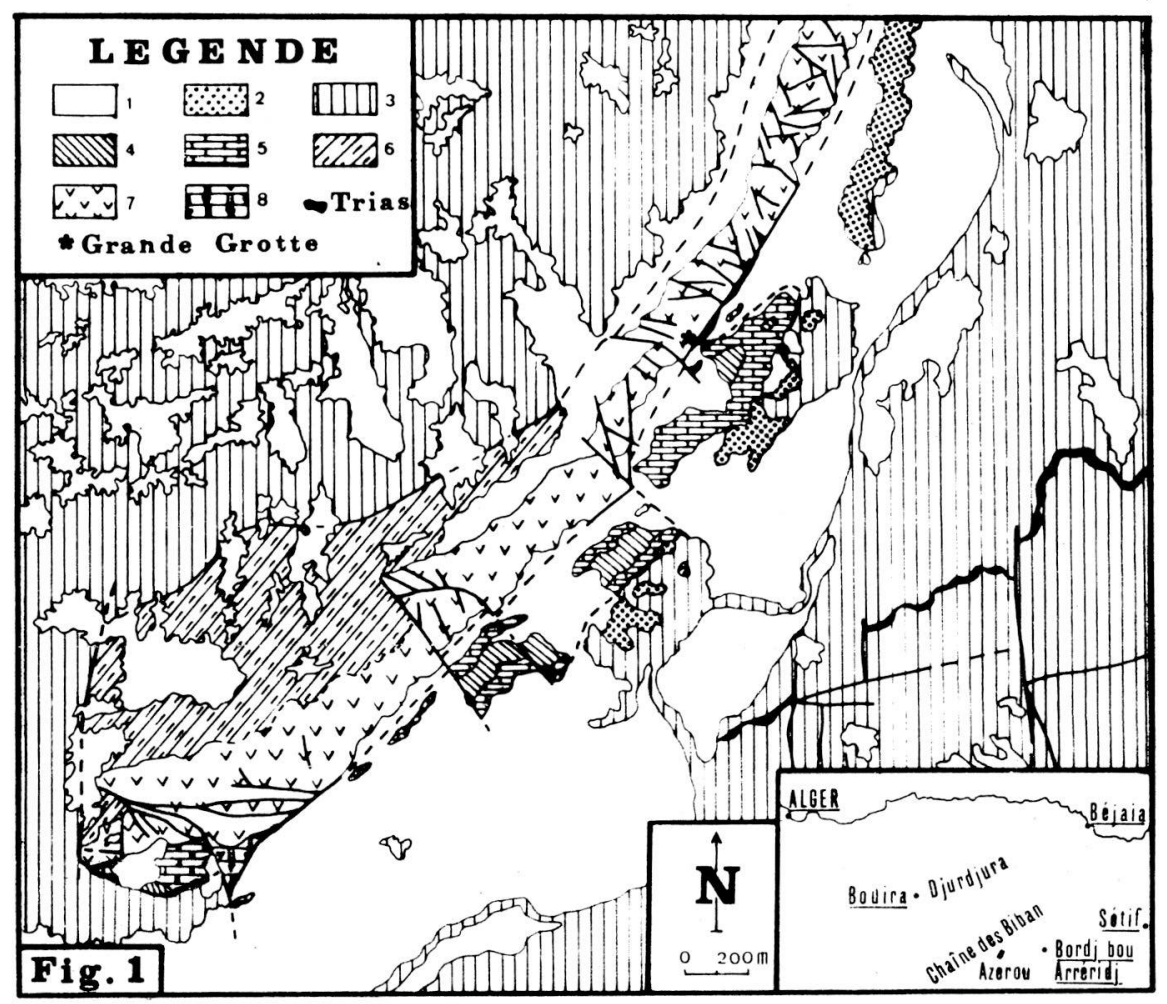




\section{LES FAITS OBSERVÉS}

\section{A. L'anticlinal de nappes}

La projection polaire des pendages des couches yprésiennes formant un replis anticlinal bordant l'Azérou el Kébir au sud-est met en évidence la nature double de cette structure. Elle est due à la superposition à un pli alpin d'axe $\mathrm{N} 90^{\circ}$ $\mathrm{E}-40^{\circ} \mathrm{E}$, d'une structure "atlasique», d'axe $\mathrm{N} 36^{\circ} \mathrm{E}-14^{\circ} \mathrm{NE}$, liée au rejeu du substratum des nappes,postérieurement à la mise en place de celles-ci. C'est au cours d'une telle phase «atlasique» récente que l'Azérou el Kébir a percé sa couverture tectonique.

Remarquons, à ce sujet, que l'anticlinal en question ne peut être attribué à une sortie en oblique de l'Azerou. D'abord, le pli n'est pas conique mais cylindrique. De plus, son axe a un plongement bien trop faible $\left(14^{\circ} \mathrm{N}\right.$.E) et sans rapport avec les stries observées sur le miroir de la faille limitant l'Azerou au S-E et plongeant à $45-55^{\circ}$ au S.W.

\section{B. La fracturation dans l'allochtone}

L'étude de la fracturation de la barre éocène armant l'anticlinal déjà évoqué, nous en a révélé sa complexité, en liaison avec son histoire géologique! Le groupe dominant de fractures A-A' (Fig. 3) est orienté $\mathrm{N} 90^{\circ}-100^{\circ} \mathrm{E}$, un second: $\mathrm{B}-\mathrm{B}^{\prime}$ à $\mathrm{N} 150^{\circ} \mathrm{E}$, et un troisième, de moindre importance, $\mathrm{C}-\mathrm{C}$ à $\mathrm{N} 10^{\circ}$ $20^{\circ}$ E. D'autres ensembles, mais moins bien individualisés pourraient éventuellement être distingués (groupe $\mathrm{D}$ à $\mathrm{N} 110^{\circ}-120^{\circ} \mathrm{E}$, groupe $\mathrm{E}$ à $\mathrm{N} 50^{\circ}-60^{\circ}$ $\mathrm{E}$ etc,...). Nous n'avons considéré que des groupes de fractures verticales ou subverticales qui seules s'organisent.

Devant la complexité de la fracturation, nous n'avons pu la corréler avec l'histoire géologique du pli (phases alpines et «atlasique»).

\section{La fracturation de l'Azerou el Kébir}

Les fractures de l'Azerou el Kébir sont excessivement dispersées (Fig. 3). Trois ensembles de fractures verticales ou subverticales s'individualisent néanmoins: un groupe $\mathrm{J}-\mathrm{J}^{\prime}$, orienté $\mathrm{N}$ 90-100 ${ }^{\circ} \mathrm{E}$, un groupe $\mathrm{K}-\mathrm{K}^{\prime}\left(\mathrm{N} 110-120^{\circ} \mathrm{E}\right)$ et, à titre accessoire, un groupe $\mathrm{L}\left(\mathrm{N} 60^{\circ} \mathrm{E}\right)$.

Si ces groupes $\mathrm{J}, \mathrm{K}$ et $\mathrm{L}$ peuvent être parallèlisés aux groupes $\mathrm{A}, \mathrm{D}$ et $\mathrm{E}$, et si, partant, la fracturation de l'Azerou el Kébir et celle de l'allochtone ont une certaine parenté, chacune n'en a pas moins son originalité: grand développement relatif des groupes $\mathrm{K}-\mathrm{K}$ ' et $\mathrm{L}$ de l'Azerou par rapport aux groupes $\mathrm{D}$ et $\mathrm{E}$ de l'allochtone, absence d'équivalents des groupes B et C. Ces différences, ainsi que la dispersion de la fracturation, s'expliquent fort bien par la complexité de l'histoire géologique (L'Azerou el Kébir est en série inverse). 


\section{RELATIONS ENTRE KARSTIFICATION ET FRACTURATION}

La grande grotte (Fig. 2) exploite préférentiellement 2 directions ( $50^{\circ}$ et $\mathrm{N}$ $\left.145^{\circ} \mathrm{E}\right)$, une troisième de façon accessoire $\left(\mathrm{N} 110^{\circ} \mathrm{E}\right)$ et une quatrième $\left(\mathrm{N} 90^{\circ}\right.$ E). La première de ces directions se retrouve dans la fracturation (groupe $\mathrm{L}$ ); c'est d'ailleurs celle de la faille bordière. La seconde ne correspond à aucun groupe préférentiel des fractures de l'Azerou mais au groupe B des fractures de l'anticlinal yprésien. Quant à la troisième, pourtant secondaire, elle correspond au groupe prépondérant des fractures de l'Azerou (groupe K). La quatrième direction, enfin, est corrélable au groupe J-J'. Ainsi, on constante que les directions structurales sont exploitées par la karstification sans lien proportionnel par rapport à leur importance statistique. L'analyse microtectonique de la seule fracturation ne permet pas de distinguer ces fractures guidant la karstification, fractures qualitativement importantes, mais noyées dans le grand nombre des cassures.

Compte tenu de l'extension speciale de la grotte étudiée et de sa position dans le contexste régional du réseau hydrogéologique, on peut exclure ici un «choix» de direction imposé par la géométrie de l'écoulement au sein du réseau. Ce choix est donc ici guidé par des critères d'ordre mécanique (fractures en détente, RENAULT 1967 et GUERIN 1974).

Nous venons d'aborder l'aspect qualitatif du problème. Il existe également un aspect quantitatif. Dans l'exemple étudié, nous constatons en effet que la karstification se développe pratiquement d'une manière inversément proportionelle à l'importance statistique des fractures. Ainsi, les directions importantes pour la karstification sont exprimées par quelques grandes cassures (souvent bien visibles dans la morphologie de la grotte) accompagnées d'un très petit nombre de fractures parallèles.

\section{CONCLUSION}

L'étude de la karstification s'avère utile, voire indispensable dans des zones structuralement complexes comme celles-ci, pour le géologue abordant les phénomènes de tectonique cassante (COIFFAIT, QUINIF 1976 \& 1977). Cette approche pluridisciplinaire du problème pourra conduire à apprécier la complexité des liens existant entre les directions du réseau karstique et celles de la fracturation.

I a fracturation du massif sub-autochtone de l'Azerou el Kébir n'est pas fondamentalement différente de celle de l'allochone asoisinam, dont les structures sont dues à une phase alpine reprise par une phase "atlasique». Parmi coutes ces fractures, la karstification n'en exploite que certaines, sans lien proportionnel par rapport à leur importance statistique. I a grotle se deselonpe suivant des fractures qualitativement importantes mais quantitativement mal reprisentes. I 'itude de la karstification s'avère done complementaire de l'analyse structurale pour ilusider les problemes mecaniques de la fracturation d'une région. 


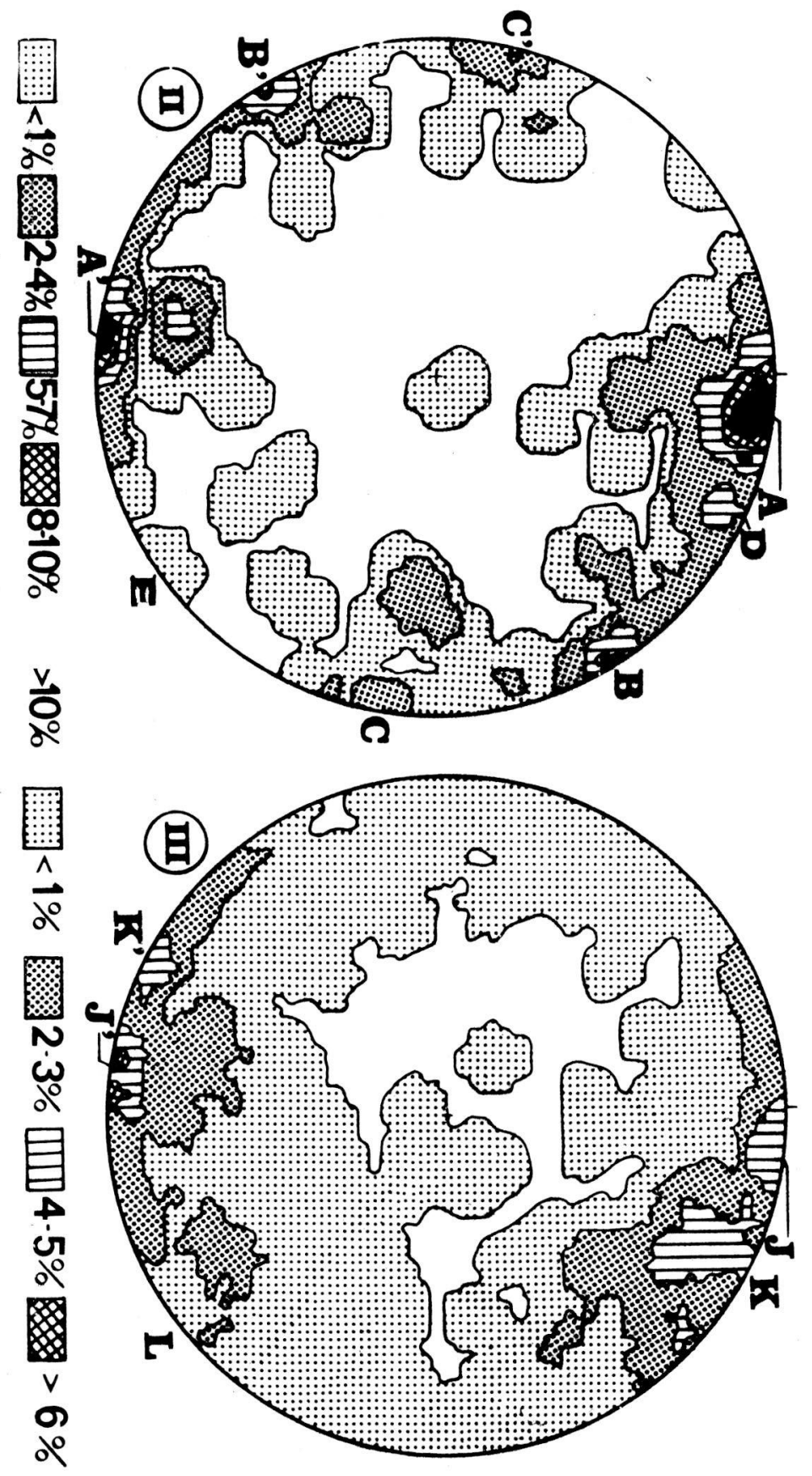




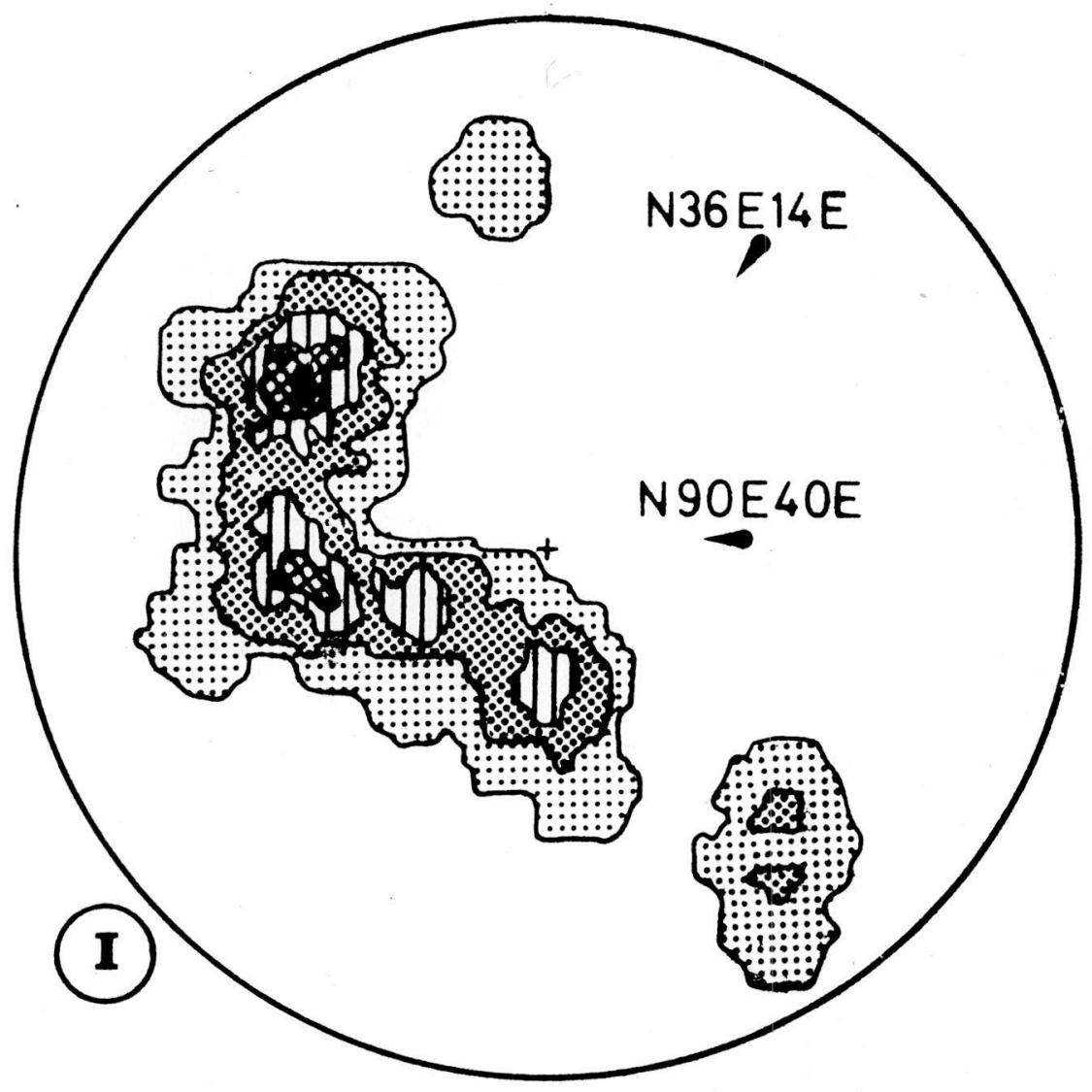

14\% $58 \%$ 向 $912 \% 13-16 \%$

\section{Fig. 3: Projections polaires:}

I. des plans de stratigraphie de l'anticlinal yprésien (allochtone);

II. des fractures de cet allochtone;

III. des fractures de l'Azerou el Kébir s.s.

Ces projections ont été étabilies grâce à l'amabilité du prof. R. FERNANDEZ RUBIO de l'Université de Grenade (Espagne) par traitement sur ordinateur des données de terrain. Toutes les projections correspondent à l'hémisphère inférieur (Canevas de Wulff). Les irtervalles de classe ont été choisis pour chaque cas en fonction d'une bonne lisibilté. 


\section{BIBLIOGRAPHIE}

CAIRE A., 1957 - Etude géologique de la chaîne des Biban. Bull. Serv. carte géol. Algérie, Nouvelle série, $\mathrm{n}^{\circ} 16,2$ tomes.

COIFFAIT P. - E. QUINIF Y. 1975 - Microformes karstiques et fracturation au Djebel Sidi R'Gheiss (Constantinois, Algérie du N.E.). Ann. Spéléo.. 30, 4, 609-618.

COIFFAIT P.-E., QUINIF Y., 1976 - La grande grotte de l'Azerou el Kébir (Algérie), Spelunca $\mathrm{n}^{\circ} 3,107-112$.

COIFFAIT P.E., QUINIF Y. 1977 - La karstification de l'Azerou - el Kébir (Algérie du Nord): Approche globale de la spéléogenese d'un massif. A paraître.

COIFFAIT P. - E., VILA J. - M., 1976 - La série renversée des Azerou (Région des Biban, Algérie) : un témoin de la plate-forme néritique sétifienne sous la nappe bibanique. C.R. Somm. Soc. Géol. Fr., fasc. 6, 269-271.

GUERIN R., 1974 - L'analyse tectonique et microtectonique dans l'étude de la karstification. Exemple des karsts du Bas-Vivarais (étude préliminaire).

Mém. et Doc. C.N.R.S., Nouvelle série, vol. 15, Phénomènes karstiques, t. 2, 81-94.

RENAULT P., 1967 - Contribution à l'étude des actions mécaniques et sédimentologiques dans la spéléogenèse. Ann. Spéléo., XXII, 209-307. 